\title{
Generic flux coupling analysis
}

\author{
Arne C. Reimers ${ }^{\mathrm{a}, \mathrm{b}, \mathrm{c}, 1, *}$, Yaron Goldstein ${ }^{\mathrm{a}, \mathrm{d}}$, Alexander Bockmayr ${ }^{\mathrm{a}, \mathrm{d}}$ \\ a Freie Universität Berlin, Arnimallee 6, Room 101, 14195 Berlin, Germany \\ ${ }^{\mathrm{b}}$ Berlin Mathematical School, Berlin, Germany \\ ' International Max Planck Research School for Computational Biology and Scientific Computing, Max Planck Institute for Molecular Genetics, Ihnestr 63-73, \\ 14195 Berlin, Germany \\ ${ }^{\mathrm{d}}$ Forschungszentrum Matheon, Technische Universität Berlin, Straße des 17. Juni 136, 10623 Berlin, Germany
}

\section{A R T I C L E I N F O}

\section{Article history:}

Received 4 August 2014

Revised 8 January 2015

Accepted 14 January 2015

Available online 22 January 2015

\section{Keywords:}

Metabolic network

Flux coupling analysis

Qualitative model

Thermodynamic constraint

\begin{abstract}
A B S T R A C T
Flux coupling analysis (FCA) has become a useful tool for aiding metabolic reconstructions and guiding genetic manipulations. Originally, it was introduced for constraint-based models of metabolic networks that are based on the steady-state assumption. Recently, we have shown that the steady-state assumption can be replaced by a weaker lattice-theoretic property related to the supports of metabolic fluxes. In this paper, we further extend our approach and develop an efficient algorithm for generic flux coupling analysis that works with any kind of qualitative pathway model. We illustrate our method by thermodynamic flux coupling analysis (tFCA), which allows studying steady-state metabolic models with loop-law thermodynamic constraints. These models do not satisfy the lattice-theoretic properties required in our previous work. For a selection of genome-scale metabolic network reconstructions, we discuss both theoretically and practically, how thermodynamic constraints strengthen the coupling results that can be obtained with classical FCA.

A prototype implementation of tFCA is available at http://hoverboard.io/L4FC.
\end{abstract}

(C) 2015 Elsevier Inc. All rights reserved.

\section{Introduction}

Constraint-based modeling has become a widely used approach for the analysis of genome-scale reconstructions of metabolic networks $[6,26]$. Given a set $\mathcal{M}$ of metabolites and a set $\mathcal{R}$ of reactions, the metabolic network is modeled by its stoichiometric matrix $S \in \mathbb{R}^{\mathcal{M} \times \mathcal{R}}$ and a set of irreversible reactions $\operatorname{Irr} \subseteq \mathcal{R}$. The sets $\mathcal{M}, \mathcal{R}$ and Irr are used as index sets, i.e., for a set $A \subseteq \mathcal{R}$ and a vector $v \in \mathbb{R}^{\mathcal{R}}, v_{A}$ denotes the sub-vector with only entries for the reactions $A$ and $S_{A}$ denotes the submatrix with only columns corresponding to the reactions in $A$. Based on this description, constraints are used to characterize the space of possible metabolic behaviors formulated using vectors $v \in \mathbb{R}^{\mathcal{R}}$ of reaction rates. The classical starting point of constraint-based modeling is the steady-state (or mass balance) constraint $S v=0$. It states that every metabolite has to be produced at the same rate as it is consumed. The set

$C=\left\{v \in \mathbb{R}^{\mathcal{R}} \mid S v=0, v_{\text {Irr }} \geq 0\right\}$

* Corresponding author at: Freie Universität Berlin, Arnimallee 6, Room 101, 14195 Berlin, Germany. Tel.: +49 3083875231; fax: +49 3083875869.

E-mail addresses: arne.c.reimers@gmail.com, arne.reimers@fu-berlin.de (A.C. Reimers), goldsteiny.de@gmail.com (Y. Goldstein),

alexander.bockmayr@fu-berlin.de (A. Bockmayr).

1 Current address: Centrum Wiskunde \& Informatica, Science Park 123, 1098 XG Amsterdam, Netherlands. Tel.: +31(0)20 5924084 is called the steady-state flux cone. It contains all flux distributions $v \in \mathbb{R}^{\mathcal{R}}$ satisfying the stoichiometric and irreversibility constraints.

A prominent example of a constraint-based method is flux balance analysis (FBA) [44]. Here, linear programming is used to predict how efficiently an organism can realize a certain biological objective, e.g., how much biomass can be produced out of a limited amount of nutrients. FBA can be used to predict the impact of gene or reaction knockouts in an organism. However, typically not all reactions in a metabolic network can carry flux independently from each other. In other words, by knocking out one reaction, one may implicitly disable flux through other reactions. Detecting this kind of dependencies is important for the identification of knock-out targets, because some reactions may be easier to manipulate than others [18]. In addition, dependency information can also be used to check the consistency of metabolic network reconstructions [7] or to find co-regulated reactions [33].

Flux coupling analysis (FCA) [8] was introduced to comprehensively analyze these kinds of dependencies between reactions. A reaction $s \in \mathcal{R}$ is called blocked if $v_{s}=0$, for all $v \in C$, otherwise $s$ is called unblocked. Burgard et al. [8] defined three types of coupling relations for a given pair of unblocked reactions $(r, s)$ :

- $s$ is directionally coupled to $r$, written $s \rightarrow r$, if for all $v \in C, v_{s} \neq 0$ implies $v_{r} \neq 0$. Equivalently, we could require that for all $v \in C$, 
$v_{r}=0$ implies $v_{s}=0$, which is denoted by $r \stackrel{=0}{\longrightarrow} s$ in Ref. [25] or [24].

- $r$ and $s$ are partially coupled, written $r \leftrightarrow s$, if both $r \rightarrow s$ and $s \rightarrow r$ hold.

- $r$ and $s$ are fully coupled, if there exists $\lambda \neq 0$ with $v_{r}=\lambda v_{s}$, for all $v \in C$.

- $r$ and $s$ are uncoupled, if neither $r \rightarrow s$ nor $s \rightarrow r$ holds.

Due to a number of theoretical and algorithmic improvements $[12,24,25]$, it is now possible to perform FCA on large genome-scale metabolic network reconstructions in a few minutes of computation time on a standard desktop computer.

As can be seen from the definition, directional coupling does not depend on the precise amount of flux through a given reaction. It only matters whether there is a flux different from zero or not. In this sense, FCA is a qualitative method. As we will see, FCA can also be applied to more general qualitative models of metabolic networks such as those introduced in Refs. [9,41]. These models do not use the steady-state assumption because it turns out to be too strong for certain applications.

Goldstein et al. [17] generalized FCA to constraint-based models that do not have to satisfy the steady-state assumption, but instead fulfill certain lattice-theoretic properties related to the supports of the metabolic fluxes. Given a flux vector $v \in \mathbb{R}^{\mathcal{R}}$, its support is defined by

$\operatorname{supp}(v):=\left\{i \in \mathcal{R} \mid v_{i} \neq 0\right\}$.

By working with $\operatorname{supp}(v)$ instead of $v$, flux coupling analysis can be performed in a qualitative way. While lattices are a very general concept [11], we have to consider here only finite lattices $L$ with reaction sets $a \subseteq \mathcal{R}$ as elements. Let $2^{\mathcal{R}}$ denote the power set of $\mathcal{R}$. A family of reaction sets $L \subseteq 2^{\mathcal{R}}$ defines a lattice if $\emptyset \in L$ and if $L$ is union-closed, i.e., $a, b \in L$ implies $a \cup b \in L$. Note that each reaction set $a \in L$ can be naturally interpreted as a metabolic pathway consisting exactly of the reactions $r \in a$. For standard FCA, the flux lattice $L^{C}$ is defined by the supports of the steady-state flux vectors,

$L^{C}:=\left\{\operatorname{supp}(v) \mid S v=0, v_{\text {Irr }} \geq 0\right\}$.

Given an arbitrary lattice $L \subseteq 2^{\mathcal{R}}$, we generalize the notion of flux coupling in the following way [17]. A reaction $s \in \mathcal{R}$ is blocked in $L$ if $s \notin a$, for all $a \in L$, otherwise $s$ is unblocked in L. Given two reactions $r$ and $s$ that are unblocked in $L$, we say that $s$ is directionally coupled in $L$ to $r$ if and only if

$\forall a \in L: s \in a \Rightarrow r \in a$.

In the case of $L=L^{C}$, this corresponds exactly to standard FCA as introduced before.

The generalization of FCA to lattices already allows analyzing a wide range of metabolic network models. For example, we may use lattices to perform FCA on constraint-based models with lower and upper bounds on the flux rates [17]. However, there are interesting cases where the lattice assumption is still too strong. Consider the network in Fig. 1. Here, reaction (1) is not directionally coupled to reaction (3), because $v=(0,0,1,1)$ is a steady-state flux. However, this flux is an internal circulation, which violates the second law of thermodynamics. As observed by Beard et al. [3], a thermodynamically feasible flux must not contain any internal circulation (also referred to as the loop-law).

If we add thermodynamic constraints, reaction (3) becomes fully coupled to (1) and thus, we get a stronger result than without these constraints.

We remark that many works on thermodynamic constraints integrate information on equilibrium constants and metabolite concentrations $[5,10,14,15,19,21,23,27,31,48]$. While the method that we present in this paper is generic and can easily be extended to such models, we here restrict ourselves for simplicity reasons to loop-law

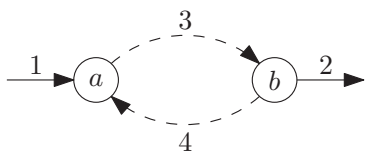

Fig. 1. Here, all reactions are irreversible. Without thermodynamic constraints, reaction (1) is not directionally coupled to reaction (3), since reactions (3) and (4) form an internal circuit (dashed arrows). With thermodynamic constraints, reactions (1) and (3) are fully coupled.

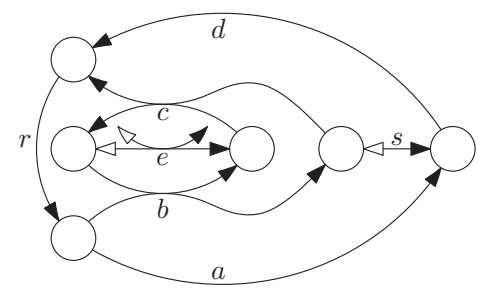

Fig. 2. The only exchange reaction in this network is $e$ and the white arrow-heads indicate reversible reactions. If $r$ carries thermodynamically feasible flux, then $s$ must also carry flux. But, $s$ can carry both positive and negative flux.

thermodynamic constraints. Loop-law thermodynamic constraints have the advantage that they do not require data on equilibrium constants and metabolite concentrations. Therefore, they have also been widely studied in the literature $[3,13,16,20,28,30,32,34,35,38,47,49]$

The key property of lattices is that metabolic pathways can be combined by taking the union of the supports. However, if the pathways have to satisfy thermodynamic constraints, this is not always possible. For example, in Fig. 2, the pathways $\{r, b, s, d, e\},\{r, a, s, c, e\}$ are thermodynamically feasible, but the combination $\{r, a, b, s, c, d, e\}$ is not thermodynamically feasible, since it contains the internal circuit $\{r, a, d\}$.

In this paper, we show how to overcome this problem. We develop an efficient algorithm for generic flux coupling analysis (generic FCA), where we do not require the lattice-theoretic axioms. As a concrete instance of our framework, we present thermodynamically constrained flux coupling analysis (tFCA) and apply it to a number of genome-scale metabolic network reconstructions.

\section{Generic FCA}

The definition of directional coupling in (1) could also be applied to the family $T \subseteq 2^{\mathcal{R}}$ of supports of thermodynamically feasible fluxes (see Section 3 for a formal definition). However, as shown in Section 1, $T$ need not be a lattice, and thus we cannot directly use the results of Ref. [17].

\subsection{FCA for arbitrary pathway models $P \subseteq 2^{\mathcal{R}}$}

In this section, we present a generic form of FCA that works not only for the supports $T \subseteq L^{C}$ of the thermodynamically feasible fluxes, but for any family $P$ of pathways that satisfies $\emptyset \neq P \subseteq 2^{\mathcal{R}}$. We call $P$ a pathway model and it can easily be seen that for any kind of qualitative metabolic network model that admits at least one feasible pathway such a pathway model exists. Furthermore, we observe on the example of elementary modes [40] that the number of pathways (the size of $P$ ) can be huge $[43,45]$. Therefore, we are not interested in computing $P$ explicitly, but we will work with $P$ implicitly to analyze the following properties:

$s$ blocked in $P: \Leftrightarrow \forall a \in P: s \notin a$, and

$$
r \stackrel{=0}{\longrightarrow} s \text { in } P: \Leftrightarrow \forall a \in P: r \notin a \Rightarrow s \notin a .
$$


To mathematically derive our results, we define the set of irreducible elements in $P$

$\mathcal{J}(P):=\left\{b \in P \backslash\{\emptyset\} \mid \forall A \subseteq P: b=\bigcup_{a \in A} a \Rightarrow b \in A\right\}$,

and the set $\mathcal{M}(P)$ of (non-trivial) minimal elements in $P$

$\mathcal{M}(P):=\{e \in P \backslash\{\emptyset\} \mid \forall a \in P: a \subsetneq e \Rightarrow a=\emptyset\}$.

Note that $\mathcal{M}(P) \subseteq \mathcal{J}(P)$, but in general not $\mathcal{M}(P)=\mathcal{J}(P)$. Although the set of minimal resp. irreducible elements of $P$ is smaller than $P$ itself, the number of minimal resp. irreducible elements (in the case of $L^{C}$ these are the elementary modes) is typically very large. Therefore, we will also avoid computing these kinds of sets.

The closure of $P$ is defined by

$\langle P\rangle=\left\{\bigcup_{a \in A} a \mid A \subseteq P\right\}$.

It is easy to see that $\langle P\rangle$ is the smallest lattice that contains $P$. We say $P$ is a generator of $\langle P\rangle$. We observe that for any lattice $L, \mathcal{J}(L)$ is the unique minimal generator, thus $L=\langle\mathcal{J}(L)|$. It follows $\langle\mathcal{J}(P)\rangle=\langle P\rangle$ and $\mathcal{J}(P)=\mathcal{J}(\langle P\rangle)$. Now we can state our first result:

Theorem 1. Consider any pathway model $\emptyset \neq P \subseteq 2^{\mathcal{R}}$ and let $B \subseteq 2^{\mathcal{R}}$ be a pathway model such that $\mathcal{J}(P) \subseteq B \subseteq\langle P\rangle$. For a reaction $s \in \mathcal{R}$, the following are equivalent:

(a) s blocked in $P$,

(b) $s$ blocked in $\langle P\rangle$,

(c) $s$ blocked in $B$,

(d) s blocked in $\langle B\rangle$.

For two reactions $r$, s that are unblocked in $P$, the following are equivalent:

(a) $r \stackrel{=0}{\longrightarrow} \sin P$,

(b) $r \stackrel{=0}{\longrightarrow} \sin \langle P\rangle$,

(c) $r \stackrel{=0}{\longrightarrow} \sin B$,

(d) $r \stackrel{=0}{\longrightarrow} \sin \langle B\rangle$.

Proof. Since $\mathcal{J}(P) \subseteq B \subseteq\langle P\rangle$, we have $\langle P\rangle=\langle B\rangle$. Thus, it is sufficient to prove (c) $\Leftrightarrow(\mathrm{d})$.

$\Rightarrow$ : Assume $s$ is unblocked (resp. $s$ is not directionally coupled to $r$ ) in $\langle B\rangle$. By definition, there exists $a \in\langle B\rangle$ such that $s \in a$ (resp. $s \in a \not \supset r)$. Since $\mathcal{J}(|B|)$ is a generator of $\langle B\rangle$, there exists $b \in \mathcal{J}(|B|)$ with $s \in b$ (resp. $s \in b \not \supset r$ ). Since $b \in\langle B\rangle$ and by definition of $\langle B\rangle$, there exists $A \subseteq B \subseteq\langle B\rangle$ with $b=\bigcup_{a \in A} a$. Since $b$ is irreducible, it follows that $b \in A$ and thus $b \in B$. This proves that $s$ is unblocked (resp. $s$ is not directionally coupled to $r$ ) in $B$.

$\Leftarrow$ : Assume $s$ is unblocked (resp. $s$ is not directionally coupled to $r$ ) in $B$. Then there exists $a \in B$ such that $s \in a$ (resp. $s \in a \not \ngtr r$ ). Since $B \subseteq\langle B\rangle$, we have $a \in\langle B\rangle$. It follows that $s$ is unblocked (resp. $s$ is not directionally coupled to $r$ ) in $\langle B\rangle$.

\subsection{FCA-algorithm for generic pathway models $P$}

In Ref. [17], we presented a generic algorithm for finding blocked reactions and determining flux coupling pairs, which works for arbitrary lattices $L \subseteq 2^{\mathcal{R}}$. In order to use this algorithm in a particular lattice $L$, we only have to provide a method test $(s)(\operatorname{resp}$. test $(r, s)$ ) that returns a lattice element $a \in L$ with $s \in a$ (resp. $s \in a \not \supset r$ ), if such elements exist, and $\emptyset$ otherwise. Here we show that the same algorithm works for flux coupling analysis for generic pathway models $P$.

Theorem 2. For a pathway model $P$ let testBlocked $: \mathcal{R} \rightarrow 2^{\mathcal{R}}$ be a function satisfying

testBlocked $_{P}(s)= \begin{cases}a, & \text { if } \exists a \in P: s \in a, \\ \emptyset, & \text { otherwise. }\end{cases}$
Let test Coupled $: \mathcal{R} \times \mathcal{R} \rightarrow 2^{\mathcal{R}}$ be a function satisfying

testCoupled $_{P}(r, s)= \begin{cases}a, & \text { if } \exists a \in P: s \in a \not \nexists r, \\ \emptyset, & \text { otherwise. }\end{cases}$

Then the algorithm introduced in Ref. [17] implemented using the functions testBlocked $d_{P}$ and testCoupled $d_{P}$ performs FCA for P.

Proof. By definition of testCoupled $P$ and testBlocked $P$ and Theorem 1 we get that

testCoupled $_{P}(r, s)=\emptyset \quad \Leftrightarrow r \stackrel{=0}{\longrightarrow} s$ in $P \quad \Leftrightarrow r \stackrel{=0}{\longrightarrow} s$ in $\langle P\rangle$

$\operatorname{blocked}_{P}(r)=\emptyset \quad \Leftrightarrow r$ blocked in $P \quad \Leftrightarrow r$ blocked in $\langle P\rangle$

Since $P \subseteq\langle P\rangle$ it follows that testCoupled $P$ and testBlocked $P$ satisfy

$\operatorname{testBlocked}_{P}(s)= \begin{cases}a, & \text { if } \exists a \in\langle P\rangle: s \in a, \\ \emptyset, & \text { otherwise }\end{cases}$

$\operatorname{testCoupled}_{P}(r, s)= \begin{cases}a, & \text { if } \exists a \in\langle P\rangle: s \in a \not \supset r, \\ \emptyset, & \text { otherwise. }\end{cases}$

Since $\langle P\rangle$ is a lattice, we can apply the algorithm introduced in Ref. [17] using testCoupled $P$ and testBlocked . $_{\text {. }}$

\section{Thermodynamic constraints}

Now we apply the framework from Section 2 to develop an algorithm for thermodynamic FCA (tFCA). We will work with the relaxed form of thermodynamic constraints introduced by Beard et al. [3], who use the following formulation:

$$
\begin{array}{rlr}
S v & =0, & \text { steady-state } \\
v_{\text {Irr }} & \geq 0, & \text { irreversible reactions } \\
\Delta \mu_{i} v_{i} & <0 \vee v_{i}=0, \forall i \in \mathcal{I}, & \text { thermodynamic constraint } \\
\Delta \mu & =\mu^{T} S_{\mathcal{I}}, & \text { potential differences } \\
v & \in \mathbb{R}^{\mathcal{R}}, \mu \in \mathbb{R}^{\mathcal{M}}, &
\end{array}
$$

where $\mathcal{I}$ denotes the set of internal reactions (i.e., reactions that are not exchange reactions), $S_{\mathcal{I}}$ the submatrix of the stoichiometric matrix $S$ corresponding to the reactions in $\mathcal{I}$, and $\mu$ the chemical potential of each metabolite (Gibbs free energy of formation). Given a metabolic network $\mathcal{N}=(\mathcal{M}, \mathcal{R}, \mathcal{I}, S$, Irr $)$, a flux vector $v \in \mathbb{R}^{\mathcal{R}}$ is called thermodynamically feasible if there exists a vector $\mu \in \mathbb{R}^{\mathcal{M}}$ such that (2), (3), (4), and (5) are satisfied.

By multiplying $\mu^{T}$ from the left side with $S_{\mathcal{I}}\left(\cdot{ }^{T}\right.$ denotes transposition), the potential differences for internal reactions are obtained. This is equivalent to the often found formulation $K \Delta \mu=0$, where $K$ is the null-space matrix of $S_{\mathcal{I}}$. The motivation behind (4) is that normally a chemical reaction carries flux if and only if it reduces Gibbs free energy $[2,4,36]$. Since many reactions are catalyzed by enzymes, however, it can happen that an enzyme, for example because of regulatory control, is not present; and hence, the corresponding reaction is effectively not possible and carries essentially no flux, even if there is a negative potential difference. Thus, zero flux is always allowed, too. Note that other formulations of thermodynamic constraints (for example in Ref. [16]) do not have this property.

As it can easily be seen from the definition, thermodynamic feasibility depends only on the sign of the fluxes. This is the idea underlying the use of oriented matroids as suggested by Beard et al. [3]. Let $\operatorname{sign}(v) \in\{-, 0,+\}^{\mathcal{R}}$ denote the vector of signs of $v \in \mathbb{R}^{\mathcal{R}}$. For example, for $v=(3,5,0,-1,2)$ we have $\operatorname{sign}(v)=(+,+, 0,-,+)$. Given a sign vector $A \in\{-, 0,+\}^{\mathcal{R}}$, we will also write $A=\left(A^{+}, A^{-}\right)$with $A^{+}=\left\{r \in \mathcal{R}: A_{r}=+\right\}, A^{-}=\left\{r \in \mathcal{R}: A_{r}=-\right\}$.

Since we want to talk about fluxes contained in other fluxes, we define the following inclusion relation:

$\left(A^{+}, A^{-}\right) \subseteq\left(B^{+}, B^{-}\right)$if and only if $A^{+} \subseteq B^{+}$and $A^{-} \subseteq B^{-}$. 
Note that if all reactions are irreversible (and thus all sign vectors of feasible fluxes are non-negative), then this subset relation is equivalent to the ordinary subset relation on the support of the fluxes.

It has been shown $[3,29,32]$ that a flux $v$ is thermodynamically feasible if and only if $v$ does not contain an internal circulation, i.e., a flux vector $w \in \mathbb{R}^{\mathcal{I}} \backslash\{0\}$ with $S_{\mathcal{I}} w=0$ and $w_{\mathcal{I} \cap I r r} \geq 0$ (note that $w$ does not use any exchange reactions):

Theorem 3. Given a metabolic network $\mathcal{N}=(\mathcal{M}, \mathcal{R}, \mathcal{I}, S$, Irr $)$, a flux vector $v \in \mathbb{R}^{\mathcal{R}}$ satisfying (2) and (3) is thermodynamically feasible if and only if there is no $w \in \mathbb{R}^{\mathcal{I}} \backslash\{0\}$ with $\operatorname{sign}(w) \subseteq \operatorname{sign}\left(v_{\mathcal{I}}\right)$ and $S_{\mathcal{I}} w=0$.

Using this characterization we define the space $T$ of thermodynamically feasible fluxes as follows:

$T:=\left\{\operatorname{supp}(v): \begin{array}{c}S v=0, v_{\text {Irr }} \geq 0 \\ \nexists w \neq 0: S_{\mathcal{I}} w=0, \operatorname{sign}(w) \subseteq \operatorname{sign}(v)\end{array}\right\}$

\section{Thermodynamic FCA}

For our implementation of thermodynamically constrained FCA, we do not operate directly on the space $T$ of thermodynamically feasible fluxes. Instead, we make use of Theorem 1 and work on the space

$B:=\left\{\operatorname{supp}(v): S v=0, v_{\text {Irr }} \geq 0, c \nsubseteq \operatorname{supp}(v) \forall c \in \mathcal{C}\right\}$.

Here, $\mathcal{C}:=\mathcal{M}\left(\left\{\operatorname{supp}(w) \mid S_{\mathcal{I}} w=0, w_{\mathcal{I} \cap I r r} \geq 0\right\}\right)$ denotes the set of minimal supports of internal circulations, which are also called the internal circuits.

Since for every internal circulation $w$ there exists a $c \in \mathcal{C}$ with $c \subseteq \operatorname{supp}(w)$ and $\operatorname{sign}(w) \subseteq \operatorname{sign}(v)$ implies $\operatorname{supp}(w) \subseteq \operatorname{supp}(v)$, we get immediately $B \subseteq T$. However, $B$ can be strictly smaller than $T$. For example, we may have flux through parallel, reversible reactions in $T$. This is not allowed in $B$, because parallel, reversible reactions together form an internal circuit.

To apply Theorem 1 , we have to show that the irreducible elements of $T$ are contained in $B$. First, we note that the irreducible elements of $T$ form a subset of the elementary modes defined as

$E:=\mathcal{M}\left(L^{C}\right)=\left\{e \in L^{C} \mid \forall a \in L^{C}: a \subsetneq e \Rightarrow a=\emptyset\right\}$.

This is an immediate consequence of the next lemma.

Lemma 1. For every $v \in T$, there exist elementary modes $e_{1}, \ldots, e_{k} \in$ $E \cap T$ with $v=\bigcup_{i=1}^{k} e_{i}$.

Proof. This lemma follows directly from Lemma 4 in Ref. [29]. Alternatively, the notion of feasibility classifier as introduced by Terzer in Ref. [42] and used in the supplementary material of Ref. [22] leads to an easy proof.

Corollary 1. $\mathcal{J}(T) \subseteq E=\mathcal{M}\left(L^{C}\right)$.

Now we can state the desired result:

Proposition 1. $\mathcal{J}(T) \subseteq B$.

Proof. By Corollary 1, every $e \in \mathcal{J}(T)$ is minimal in $L^{C}$. Thus, there is no $a \in L^{C} \backslash\{\emptyset\}$ with $a \subsetneq e$. Assume $e \notin B$. Then there exists $c \in \mathcal{C}$ with $c \subseteq e$. Clearly, $c \in L^{C}$. If $c \subsetneq e$, then $e$ is not minimal in $L^{C}$. If $=\operatorname{supp}(e)$, then $e$ is an internal circuit, and it follows $e \notin T$. In both cases, we get a contradiction, hence $e \in B$.

Altogether, we have shown $\mathcal{J}(T) \subseteq B \subseteq T \subseteq\langle T\rangle$. According to Theorem 1 , we may perform FCA in $T$ via FCA in $B$. Therefore, we define $\operatorname{methods~testBlocked~}_{B}(s)$ and testCoupled ${ }_{B}(r, s)$ which we can then use by Theorem 2 in the lattice based algorithm from Ref. [17]. We remark that the problems testBlocked ${ }_{B}(s)$ and testCoupled ${ }_{B}(r, s)$ are NP-hard, because deciding if a reaction is not blocked in $T$ is NP-hard [28] (note that this complexity statement is based on the fact that we allow irreversibility constraints on internal reactions). To solve these NP-hard problems exactly, we use a mixed integer linear program (MILP) in which we solve (for sufficiently large $M>0$ ):

$\min 0$

s.t. $\quad S v=0$,

$v_{\text {Irr }} \geq 0$,

$-M a_{i} \leq v_{i} \leq M a_{i}$,

$\sum_{i \in C} a_{i} \leq|c|-1, \quad \forall c \in \mathcal{C}$

$v_{s}=2 b-1, v_{s} \in\{-1,1\}$,

$v_{r}=0$,

$a_{i}, b \in\{0,1\} \quad \forall i \in \mathcal{R}$.

Here, $|c|$ is the cardinality of $c \in \mathcal{C}$. The idea of this MILP is the following. The variables $a_{i}$ describe the support of the flux vector $v$. In (8), we require only that $a_{i}=0$ implies $v_{i}=0$, because the $a_{i}$ appear only in the circuit constraints (9). Violated circuit-constraints cannot become feasible by setting additional $a_{i}=1$. The $0-1$ variable $b$ is used to force positive or negative flux through reaction $s$.

The functions testBlocked $(s)\left(\right.$ resp. testCoupled $\left.{ }_{B}(r, s)\right)$ can now be implemented by searching for a feasible solution $v$ of the MILP (6)-(10) (resp. (6)-(11)). If this MILP is infeasible, we return the value $\emptyset$, otherwise the reaction set $a=\operatorname{supp}(v)$. Optimization is not needed, which is why the objective function is simply the constant 0 .

\section{Implementation}

The efficiency of the algorithm in Ref. [17] results from a search via nested intervals. The unblocked reactions in a lattice $L$ are exactly those contained in the maximum element

$\max :=1_{L}:=\bigcup_{a \in L} a$.

Similarly, the reactions not coupled to $r$ in $L$ are exactly those contained in the maximum element

$\max _{r}:=1_{L_{\perp\{r\}}}:=\bigcup_{a \in L_{\perp\{r\}}} a$

of the lattice

$L_{\perp\{r\}}=\{a \in L \mid r \notin a\}$.

The algorithm in Ref. [17] determines max (resp. $\max _{r}$ ) via lower and upper bounds $\mathrm{lb} \subseteq \max \subseteq$ ub (resp. $\mathrm{lb} \subseteq \max _{r} \subseteq$ ub). The lower bound $\mathrm{lb}$ is the union of known pathways ("witnesses"), which in the case of $\max _{r}$ must not contain $r$. The upper bound ub excludes reactions that are known to be blocked. Thus, only the remaining reactions $s \in \mathrm{ub} \backslash \mathrm{lb}$ have to be tested. Traditional FCA tests the feasibility of

$\left\{v \in \mathbb{R}^{\mathcal{R}}\left|S v=0, v_{\text {Irr }} \geq 0, v_{r}=0,\right| v_{S} \mid \geq 1\right\}$

via linear programming (LP). Supports of feasible solutions extend the lower bound (by at least adding $s$ ), while infeasibility leads to an update $\mathrm{ub} \leftarrow \mathrm{ub} \backslash\{s\}$.

In principle, it would be possible to realize thermodynamic FCA just by replacing these feasibility tests with the functions testBlocked $_{B}$ and testCoupled ${ }_{B}$ introduced in Section 4. However, when calling these functions, we have to solve an NP-hard problem due to the thermodynamic constraints [28]. Since solving the MILP is computationally hard, we decided to use our knowledge of the lattice structure to minimize the number of function calls. We introduce a relaxation for preprocessing, which is $\langle T\rangle \subseteq L^{C}$. For the search of unblocked reactions in $T$ (note $1_{T}=1_{\langle B\rangle}$ ), we start with ub $=1_{L^{C}}$. To find directionally coupled reactions in $T$ (derived from $1_{\langle B\rangle_{\perp\{r\}}}$ ), we continue with ub $=1_{T} \cap 1_{L_{\perp\{r\}}^{C}}$, where $1_{L_{\perp\{r\}}^{C}}$ is the set of reactions not directionally coupled to $r$ by traditional FCA. In Appendix A, we 


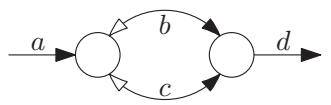

Fig. 3. Example with uncoupled reactions that are thermodynamically coupled. The white arrow-heads indicate that the reactions are reversible.

describe an example run of our algorithm on the network shown in Fig. 2.

Our software has been implemented in Java and alternates between traditional FCA and TFCA, using the results of FCA computations whenever possible to infer tFCA properties. To perform traditional FCA and the test testCoupled ${ }_{B}$, we use Cplex 12.5 for solving the LPs and MILPs. The internal circuits of the network are computed with a variant of the WW-algorithm [46] using the efmtool by Terzer et al. [42]. All the networks analyzed in this study have a low number of internal circuits, which made this approach feasible and easy to implement.

A prototype implementation of tFCA is available at http:// hoverboard.io/L4FC.

\section{Discussion}

\subsection{Theoretical differences}

\subsubsection{Standard couplings are preserved}

If $r \stackrel{=0}{\longrightarrow} s$ in $L^{C}$, then $r \stackrel{=0}{\longrightarrow} s$ in $T$, since an infeasible system cannot become feasible by adding constraints. It follows that if two reactions are directionally coupled in $L^{C}$, they are also directionally coupled in $T$.

\subsubsection{New partial couplings}

In Fig. 2, we see an example where $r \stackrel{=0}{\longrightarrow} s$ in $L^{C}$, but $r$ is not directionally coupled to $s$ in $L^{C}$ (and thus $r$ is not partially coupled to $s$ in $L^{C}$ ). However, $s \stackrel{=0}{\longrightarrow} r$ in $T$, hence $r$ is partially coupled to $s$ in $T$. Further examples are the pairwise in $L^{C}$ uncoupled reactions $a, b, c, d$. With thermodynamic constraints however, $a \leftrightarrow c$ in $T$ and $b \leftrightarrow d$ in $T$. In particular, we can deduce $v_{a}=v_{c}$ and $v_{b}=v_{d}$ for every thermodynamically feasible flux vector.

\subsubsection{New directional couplings}

In Fig. 3, we see an example where reactions $a, b$ are uncoupled in $L^{C}$ (because of flux vectors with supports $\{a, c, d\}$ and $\{b, c\}$ ). But, $b$ is directionally coupled to $a$ in $T$, since flux through the circuit $\{b, c\}$ alone is thermodynamically infeasible.

\subsection{Practical comparison}

To evaluate our method in practice, we compared standard FCA and tFCA on several genome-scale metabolic network reconstructions from the BiGG-database [39]. The computational experiments were done with Java Oracle JDK 1.7.45 on a MacBook Air (2012), $1.8 \mathrm{GHz}$ Intel Core i5, 4GB RAM, Mac OS X 10.8. For solving the linear programs and MILPs, we used CPLEX 12.5. The results are given in Table 1.

In all the considered networks, tFCA was able to detect additional blocked and coupled reactions. However, the impact of the additional thermodynamic constraints heavily depends on the network.

When we analyze all pairs of coupled reactions, there is a lot of redundant information. By transitivity, if we have couplings $a \stackrel{=0}{\longrightarrow}$ $b, b \stackrel{=0}{\longrightarrow} c, a \stackrel{=0}{\longrightarrow} c$, then the third can be inferred from the first and the second. In practice, this can lead to a quadratic blow-up of redundant couplings and hence the number of coupled pairs does not really reflect the gained information. In order to get a more adequate description, we computed a minimum set of couplings from which all other couplings can be deduced (also called a transitive reduction [1]).
Table 1

Comparison of thermodynamic flux coupling results for different genome-scale networks.

\begin{tabular}{llrrrr}
\hline Model & & Blocked & Couples & Runtime & Pre-processing \\
\hline E. coli iAF1260 & FCA & 839 & 2101 & 36.35 & \\
& tFCA & 848 & 2128 & 47.74 & 19.87 \\
& Extension & 9 & 49 & & \\
S. cerevisiae iND750 & FCA & 635 & 885 & 7.73 & \\
& tFCA & 640 & 935 & 12.43 & 4.74 \\
M. tuberculosis iNJ661 & Extension & 5 & 58 & & \\
& FCA & 281 & 831 & 6.08 & \\
& tFCA & 287 & 834 & 9.46 & 3.26 \\
S. aureus iSB619 & Extension & 6 & 9 & & \\
& FCA & 278 & 544 & 3.02 & \\
& tFCA & 279 & 546 & 3.95 & 1.04 \\
H. pylori ilT341 & Extension & 1 & 3 & & \\
& FCA & 118 & 516 & 2.2 & \\
& tFCA & 124 & 515 & 5.05 & 0.81 \\
& Extension & 6 & 10 & & \\
\hline
\end{tabular}

Blocked: Number of blocked reactions in the network. Couples: Minimal number of pairs of directionally coupled reactions $r \stackrel{=0}{\longrightarrow} s$ from which all couplings can be induced by transitive closure. Runtime: Total runtime without pre-processing (calculation of internal circuits for tFCA). Times are given in seconds. Pre-processing: Time spent on pre-processing in seconds. FCA: Results for traditional FCA (steady-state assumption). tFCA: Results for thermodynamical FCA (steady-state, no internal circuits). Extension: Minimal number of changes necessary to extend the FCA coupling graph to the tFCA coupling graph.

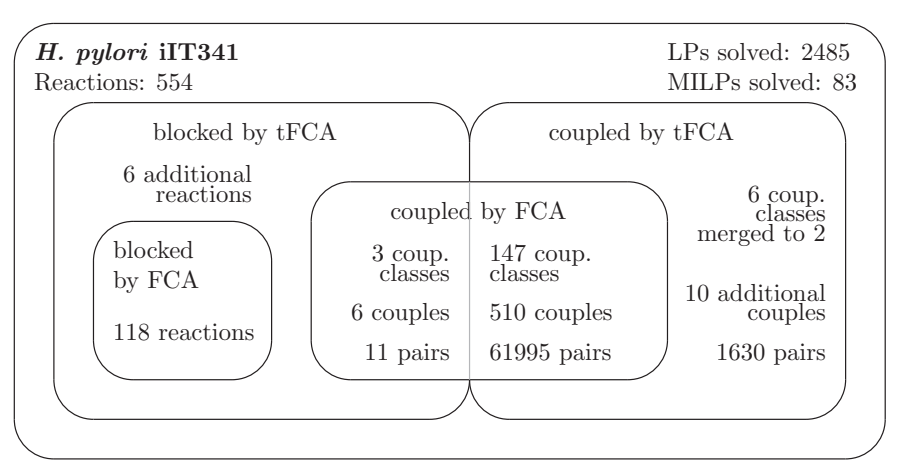

Fig. 4. Results for $H$. pylori iIT341. We found that homoserine O-trans-acetylase (HSERTA), O-acetyl-L-homoserine succinate-lyase (adding cysteine) (METB1r) and 0 succinylhomoserine lyase (SHSL1r, SHSL2r) are not only necessary for biomass production (as computed by standard FCA) but cannot work without this function. Together with the biomass reaction and all its partially coupled reactions they form one of the new coupling classes. Furthermore, the under tFCA partially coupled (but not fully coupled) SHSL2r and HSERTA were originally (in FCA) uncoupled. "couples" refers to the minimal number of (additional) couplings from which all couplings can be inferred by transitive closure.

Note however, that even this can lead to weird looking results as in the case of $H$. pylori iT341, where the number of generating couplings decreases. This is caused by the fact that some couplings involve reactions that become blocked using thermodynamic constraints (see also Fig. 4) and thus are not counted as couplings anymore in the tFCA case. Also it can happen that the addition of couplings allows the implicit deduction of old couplings and thus also reduce the size of a minimal generator. Therefore, we compute a minimum set of tFCAcouplings that together with all FCA-couplings can be used to deduce all tFCA couplings. The details of this computation are described in Ref. [37].

In average, we found around 1-6\% of additional couplings (in the minimal extension). In most cases, new directional couplings arose from previously uncoupled reactions. Only in one case, previously uncoupled reactions became partially coupled. We also observed that the reaction cystathionine g-lyase (CYSTGL) was part of new directional 
couplings in $M$. tuberculosis, while in S. cerevisiae the thermodynamic constraints blocked this reaction.

In the case of $H$. pylori iIT341, several new couplings are found because these reactions are part of the main biomass production pathway. If one of the reactions in the pathway is blocked, the whole pathway breaks down, which induces these couplings. However, if some reaction is part of an internal circuit, standard FCA allows such a reaction to be active via the circuit. This way standard FCA cannot detect that the reaction is part of the pathway. The reactions homoserine $\mathrm{O}$-trans-acetylase (HSERTA), $\mathrm{O}$-acetyl-L-homoserine succinate-lyase (adding cysteine) (METB1r) and O-succinylhomoserine lyase (SHSL1r, SHSL2r) in H. pylori iIT341 provide an example for this effect.

A summary of the results for $H$. pylori iIT341 is given in Fig. 4 . The coupling types are depicted in a set-diagram style:

- The set of reactions blocked without thermodynamic constraints (blocked by FCA) is contained in the box of reactions blocked with thermodynamic constraints (blocked by tFCA). The number of reactions that are only blocked due to thermodynamic constraints is indicated in the set difference. In the case of $H$. pylori, there are 6 such reactions.

- Since there are more reactions blocked with thermodynamic constraints than without, some of the coupled reactions that we found by standard FCA contain reactions that are blocked with thermodynamic constraints. This is why the set of reactions coupled by FCA intersects the set of thermodynamically blocked reactions and the set of thermodynamically coupled reactions. For both intersections, we report how many pairs of reactions fall into the respective category.

- In the set difference of the thermodynamically minus the normally coupled reactions, we included the number of coupling pairs that fell into this category.

In Fig. 4, we see that the standard FCA couplings can be represented by 516 couplings, 6 of which are actually blocked by thermodynamic constraints. Thermodynamic constraints give additional information on 10 couplings. Thermodynamic constraints also merged 6 groups of partially coupled reactions to 2 groups.

Table 1 shows that the new tFCA algorithm runs only slightly slower than the FCA algorithm from Ref. [17]. This is achieved by the pre-processing step that first applies standard FCA, which already detects many couplings (see Section 6.1.1). In addition, witnesses are found that prove some reactions to be also thermodynamically unblocked, or uncoupled. Only for the few remaining cases where we cannot deduce any information from the previous step, we have to start the MILP solver.

\section{Conclusion}

In this work we extended FCA to arbitrary qualitative models, called pathway models. This removes any kind of restrictions from this analysis technique that existed previously, where the users were bound to lattice properties (which generalized the steady-state flux space).

As a show case we presented a refined version of FCA that finds more coupled reactions than standard FCA by integrating thermodynamic constraints. Although thermodynamic constraints were used that are usually NP-hard, it was possible to also analyze genomescale networks like E. coli iAF1260 in a few minutes. We observed that thermodynamic constraints do not only give additional blocked reactions but also additional coupled reactions. The concrete impact highly depends on the network that is analyzed.

The theoretical foundations presented here allow an easy extension to even more involved models with potentially much higher impact on the flux coupling results. The use of MILPs in the test methods iscoupled and isBlocked is highly flexible. For example for the integration of equilibrium constants and metabolite concentrations the MILPs could be simply replaced using formulations developed in Ref. [21].

A prototype implementation of tFCA is available at http:// hoverboard.io/L4FC.

\section{Authors contributions}

AR and YG developed the theory and analyzed the results. YG implemented the algorithm. $\mathrm{AB}$ initiated and directed the research. All authors worked on and approved the final manuscript.

\section{Acknowledgments}

We thank Alexandra Grigore for ideas on presenting the flux coupling results.

The PhD work of Arne Reimers and Yaron Goldstein was supported by the Berlin Mathematical School and the Gerhard C. Starck Stiftung.

\section{Appendix A}

Let us consider the network shown in Fig. 2. To exemplify the algorithm presented in this paper we demonstrate our method step by step. We assume that the reactions are sorted in alphabetical order and hence, we will run the coupling tests in alphabetical order. For more details see Ref. [17]. We observe that in this example network no reaction is blocked with thermodynamic constraints.

In the first step of the algorithm, we apply ordinary FCA without thermodynamic constraints, because couplings in $L^{C}$ imply couplings in $T$ (see Section 6.1.1). For each reaction $x$ we iterate through all reactions $y$ in alphabetical order and run testCoupled $L_{L}(x, y)$. In other words, we block the reaction $x$ and check if $y$ is also blocked. If $y$ is not blocked, we get a pathway that contains $y$ but not $x$. This pathway is also a witness that other reaction pairs are uncoupled. Hence, these pathways are stored and reused when applicable.

- $a$ blocked:

- $\{b, c, r\} \in L^{C}$

- $\{b, d, e, r, s\} \in L^{C}$

- $b$ blocked

- $\{a, d, r\} \in L^{C}$

- $\{a, c, d, e, r, s\} \in L^{C}$

- $c$ blocked

- $\{a, d, r\}$ reused

- $\{b, d, e, r, s\}$ reused

- $d$ blocked

- $\{a, b, c, e, r, s\} \in L^{C}$

- $e$ blocked

- $\{a, d, r\}$ reused

- $\{b, c, r\}$ reused

- $s$ is blocked

- $s$ blocked

- $\{a, d, r\}$ reused

- $\{b, c, r\}$ reused

- $e$ is blocked

- $r$ blocked

- $a, b, c, d, e$ are blocked

- $s$ is blocked by transitivity 


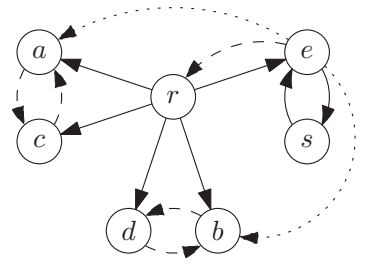

Fig. A.1. Couplings of the network from Fig. 2. An arc from node $x$ to nody $y$ means that $x \stackrel{=0}{\longrightarrow} y$. Continuous arcs are arcs derived from ordinary FCA. Dashed and dotted arcs denote additional couplings for tFCA. The dashed arcs mark a minimal extension from the FCA couplings to the TFCA couplings. Couplings that were not explicitly found by the algorithm by solving an infeasible LP or MILP, but which were computed by transitivity, are not shown.

We observe that the computed witnesses do not have to be minimal pathways, as in the case of $\{a, b, c, e, r, s\}$. Many witnesses can be reused and thus, only 12 LPs need to be solved although there are $7 \times 6=42$ possible coupling pairs. Of these $12 \mathrm{LPs}$, we find 5 LPs to be feasible, each feasible LP is giving us a solution that tells about reactions that are not coupled (without thermodynamic constraints). Seven LPs are infeasible and each of them gives us at least one coupling. Note that we do not explicitly have to check $r \stackrel{=0}{\longrightarrow} s$, because $r \stackrel{=0}{\longrightarrow} e$ and $e \stackrel{=0}{\longrightarrow} s$ have been identified beforehand and they imply this coupling by transitivity. The coupling graph is shown in Fig. A.1.

Note that the couplings we inferred from FCA are also couplings in tFCA and we do not have to recompute them. But this is not all the information that we can reuse from the FCA round. For example, the pathway $\{b, d, e, r, s\}$ is thermodynamically feasible and we can keep it as a witness.

- $a$ blocked:

- $\{b, d, e, r, s\} \in T$ reused

- $c$ is blocked

- $b$ blocked

- $\{a, c, e, r, s\} \in T$

- $d$ is blocked

- $c$ blocked

- $\{b, d, e, r, s\} \in T$ reused

- $a$ is blocked

- $d$ blocked

- $\{a, c, e, r, s\} \in T$ reused

- $b$ blocked

- e blocked

- $a$ is blocked

- $c$ is blocked by transitivity

- $b$ is blocked

- $d$ is blocked by transitivity

$-r$ is blocked

- $s$ is blocked from FCA

\section{- $s$ blocked}

- $a, b, c, d, r$ are blocked by transitivity

- $r$ blocked

- everything is already blocked from FCA

We observe that one MILP was solved to feasibility, while 7 MILPs were infeasible and gave rise to couplings under thermodynamic constraints. Using the transitivity of the coupling relation we were able to identify 9 additional couplings without solving any MILPs. Furthermore, we observe that the number of MILPs that needed to be solved could have been reduced even more by choosing a better order of the reactions and testing $e \stackrel{=0}{\longrightarrow} r$ in $T$ first, since this coupling implies $e \stackrel{=0}{\longrightarrow} a$ in $T$ and $e \stackrel{=0}{\longrightarrow} b$ in $T$ as can be seen in Fig. A. 1 .

We conclude that while the naive algorithm that would have tested all pairs would have to solve 42 MILPs, we only had to solve 8 MILPs to arrive at the same information.

\section{References}

[1] A.V. Aho, M.R. Garey, J.D. Ullman, The transitive reduction of a directed graph, SIAM J. Comput. 1 (2) (1972) 131-137.

[2] A.A. Robert, Thermodynamics of Biochemcial Reactions, Massachusetts Institute of Technology, Cambridge, MA, 2003.

[3] D.A. Beard, E. Babson, E. Curtis, H. Qian, Thermodynamic constraints for biochemical networks, J. Theor. Biol. 228 (2004) 327-333.

[4] D.A. Beard, S.d. Liang, H. Qian, Energy balance for analysis of complex metabolic networks, Biophys. J. 83 (2002) 79-86.

[5] D.A. Beard, H. Qian, Thermodynamic-based computational profiling of cellular regulatory control in hepatocyte metabolism, Am. J. Physiol. - Endocrinol. Metab. 288 (2005) E633-E644.

[6] A. Bordbar, J.M. Monk, Z.A. King, B. Palsson, Constraint-based models predict metabolic and associated cellular functions, Nat. Rev. Genet. 15 (2) (2014) 107-120.

[7] J.G. Bundy, B.A. Papp, R. Harmston, R.A. Browne, E.M. Clayson, N. Burton, R.J. Reece, S.G. Oliver, K.M. Brindle, Evaluation of predicted network modules in yeast metabolism using nmr-based metabolite profiling, Genome Res. 17 (4) (2007) 510-519.

[8] A.P. Burgard, V. Nikolaev Evgeni, C.H. Schilling, C.D. Maranas, Flux coupling analysis of genome-scale metabolic network reconstructions, Genome Res. 14 (2) (2004) 301-312.

[9] P. Carbonell, D. Fichera, S. Pandit, J.L. Faulon, Enumerating metabolic pathways for the production of heterologous target chemicals in chassis organisms, BMC Syst. Biol. 6 (1) (2012) 10.

[10] G. Cogne, M. Rügen, A. Bockmayr, M. Titical, C.G. Dussap, J.F.A.o. Cornet, J. Legrand A model-based method for investigating bioenergetic processes in autotrophically growing eukaryotic microalgae: application to the green algae, Chlamydomonas reinhardtii, Biotechnol. Prog. 27 (3) (2011) 631-640.

[11] A.D. Brian, 2002, Introduction to Lattices and Order, Cambridge University Press.

[12] L. David, S.A. Marashi, A. Larhlimi, B. Mieth, A. Bockmayr, Ffca: a feasibility-based method for flux coupling analysis of metabolic networks, BMC Bioinformatics 12 (2011) 236.

[13] D. de Martino, F. Capuani, M. Mori, A. de Martino, E. Marinari, Counting and correcting thermodynamically infeasible flux cycles in genome-scale metabolic networks, arXiv:1310.3693 [q-bio.MN].

[14] R.M.T. Fleming, I. Thiele, H.P. Nasheuer, Quantitative assignment of reaction directionality in constraint-based models of metabolism: application to Escherichia coli, Biophys. Chem. 145 (2009) 47-56.

[15] R.M.T. Fleming, I. Thiele, G. Provan, H.P. Nasheuer, Integrated stoichiometric, thermodynamic and kinetic modelling of steady state metabolism, J. Theor. Biol. 264 (2010) 683-692.

[16] R.M.T. Fleming, C.M. Maes, M.A. Saunders, Y. Ye, B.O. Palsson, A variational principle for computing nonequilibrium fluxes and potentials in genome-scale biochemical networks, J. Theor. Biol. 292 (2012) 71-77.

[17] Y.A.B. Goldstein, A. Bockmayr, A lattice-theoretic framework for metabolic pathway analysis, in: A. Gupta, T. A. Henzinger (Eds.), Computational Methods in Systems Biology, Lecture Notes in Computer Science, vol. 8130, Springer, Berlin/Heidelberg, 2013, pp. 178-191.

[18] U.U. Haus, S. Klamt, T. Stephen, Computing knockout strategies in metabolic networks, J. Comput. Biol. 15 (3) (2008) 259-268.

[19] C.S. Henry, L.J. Broadbelt, V. Hatzimanikatis, Thermodynamics-based metabolic flux analysis, Biophys. J. 92 (2007) 1792-1805.

[20] W.J. Heuett, H. Qian, Combining flux and energy balance analysis to model largescale biochemical networks, J. Bioinform. Comput. Biol. 4 (6) (2006) 1227-1243.

[21] A. Hoppe, S. Hoffmann, H.G. Holzhütter, Including metabolite concentrations into flux balance analysis: thermodynamic realizability as a constraint on flux distributions in metabolic networks, BMC Syst. Biol. 1 (23) (2007).

[22] S.J. Jol, A. Kümmel, M. Terzer, J. Stelling, M. Heinemann, System-level insights into yeast metabolism by thermodynamic analysis of elementary flux modes, PLoS Comput. Biol. 8 (3) (2012).

[23] A. Kümmel, S. Panke, M. Heinemann, Systematic assignment of thermodynamic constraints in metabolic network models, BMC Bioinformatics 7 (512) (2006).

[24] A. Larhlimi, L. David, J. Selbig, A. Bockmayr, F2c2: a fast tool for the computation of flux coupling in gen ome-scale metabolic networks, BMC Bioinformatics 13 (2012) 57.

[25] A. Larhlimi, A. Bockmayr, A new approach to flux coupling analysis of metabolic networks, Comput. Life Sci. II 4216 (2006) 205-215.

[26] N.E. Lewis, H. Nagarajan, B. Palsson, Constraining the metabolic genotypephenotype relationship using a phylogeny of in silico methods, Nat. Rev. Microbiol. 10 (4) (2012) 291-305.

[27] M.L. Mavrovouniotis, Duality theory for thermodynamic bottlenecks in bioreaction pathways, Chem. Eng. Sci. 51 (9) (1996) 1495-1507.

[28] A.C. Müller, A. Bockmayr, Fast thermodynamically constrained flux variability analysis, Bioinformatics 29 (7) (2013) 903-909. 
[29] A. Müller, Thermodynamic constraints in metabolic networks (Master's thesis), Freie Universität Berlin, Fachbereich Mathematik und Informatik, 2012.

[30] R. Nigam, S. Liang, Algorithm for perturbing thermodynamically infeasible metabolic networks, Comput. Biol. Med. 37 (2007) 126-133.

[31] E. Noor, B.E. Arren, A. Flamholz, E. Reznik, W. Liebermeister, R. Milo, Pathway thermodynamics highlights kinetics obstacles in central metabolism, PLoS Comput. Biol. (2014).

[32] E. Noor, N.E. Lewis, R. Milo, A proof for loop-law constraints in stoichiometric metabolic networks, BMC Syst. Biol. 6 (2012) 140.

[33] R.A. Notebaart, B. Teusink, R.J. Siezen, B. Papp, Co-regulation of metabolic genes is better explained by flux coupling than by network distance, PLoS Comput. Biol. 4 (1) (2008) e26

[34] N.D. Price, I. Famili, D.A. Beard, B.O. Palsson, Extreme pathways and Kirchhoff's second law, Biophys. J. 83 (2002) 2879-2882.

[35] N.D. Price, I. Thiele, B.O. Palsson, Candidate states of Helicopacter pylori's genomescale metabolic network upon application of "loop law" thermodynamic constraints, Biophys. J. 90 (2006) 3919-3928.

[36] H. Qian, D.A. Beard, Thermodynamics of stoichiometric biochemical networks in living systems far from equilibrium, Biophys. Chem. 114 (2005) 213-220.

[37] A.C. Reimers, A.M. Reimers, Y.A.B. Goldstein, Minimal equivalent subgraphs containing a given set of arcs: Technical Report \#1053, Matheon, March 2014.

[38] J. Schellenberger, N.E. Lewis, B.Ø. Palsson, Elimination of thermodynamically infeasible loops in steady-state metabolic models, Biophys. J. 100 (2011) 544-553.
[39] J. Schellenberger, J.O. Park, T.M. Conrad, B.O. Palsson, Bigg: a biochemical genetic and genomic knowledgebase of large scale metabolic reconstructions, BMC Bioinformatics 11 (2010) 213.

[40] S. Schuster, C. Hilgetag, On elementary flux modes in biochemical systems at steady state, J. Biol. Syst. 2 (1994) 165-182.

[41] T. Soh, K. Inoue, Identifying necessary reactions in metabolic pathways by minimal model generation, ECAI (2010) 277-282.

[42] M. Terzer, Large scale methods to enumerate extreme rays and elementary modes (Ph.D. thesis), Swiss Federal Institute of Technology, Zurich, 2009.

[43] M. Terzer, J. Stelling, Large-scale computation of elementary flux modes with bit pattern trees, Bioinformatics 24 (19) (2008) 2229-2235.

[44] A. Varma, B.O. Palsson, Metabolic flux balancing: basic concepts, scientific and practical use, Nat. Biotechnol. 12 (1994) 994-998.

[45] A. von Kamp, S. Schuster, Metatool 5.0: fast and flexible elementary modes analysis, Bioinformatics 22 (15) (2006) 1930-1931.

[46] J. Wright, A. Wagner, Exhaustive identification of steady state cycles in large stoichiometric networks, BMC Syst. Biol. 2 (61) (2008).

[47] L. Xie, Avoid internal loops in steady state flux space sampling, arXiv:1210.5234, 2012.

[48] F. Yang, D.A. Beard, Thermodynamically based profiling of drug metabolism and drug-drug metabolic interactions: a case study of acetaminophen and ethanol toxic interaction, Biophys. Chem. 120 (2006) 121-134.

[49] F. Yang, H. Qian, D.A. Beard, Ab initio prediction of thermodynamically feasible reaction directions from biochemical network stoichiometry, Metab. Eng. 7(2005) 251-259. 